\title{
Do we form or deform? Qualitative Investigation in Publics Hospitals of Madrid
}

Gómez Camuñas María Jesús ${ }^{1}$ *, González Villanueva Purificación ${ }^{2}$

${ }^{1}$ Health Center, Primary Care, Madrid Health Service, Madrid, Spain

${ }^{2}$ Faculty of Nursing, University of Alcalá de Henares, Madrid, Spain

\section{Email address:}

mjesusgomezc@telefonica.net (G.C., María Jesús) ${ }^{1 *}$

purificacion.gonzale@uah.es (G. V., Purificación) ${ }^{2}$ *

Corresponding author 